\title{
High-Mobility Group Nucleosome-Binding Protein 1 Mediates Renal Fibrosis Correlating with Macrophages Accumulation and Epithelial-to-Mesenchymal Transition in Diabetic Nephropathy Mice Model
}

\author{
Jiali $\mathrm{Yu}^{\mathrm{a}}$ Rong Dong ${ }^{\mathrm{a}}$ Jingjing $\mathrm{Da}^{\mathrm{a}}$ Jiayu Li ${ }^{\mathrm{b}}$ Fuxun $\mathrm{Yu}^{\mathrm{c}}$ Yan Zha ${ }^{\mathrm{a}}$ \\ a'Department of Nephrology, Guizhou Provincial People's Hospital, Guiyang, PR China; \\ ${ }^{b}$ Guizhou University School of Medicine, Guizhou Medical University, Guiyang, PR China; \\ 'Central laboratory, Guizhou Provincial People's Hospital, Guiyang, PR China
}

\section{Keywords}

Diabetic nephropathy · Fibrosis · Macrophages · Epithelial-to-mesenchymal transition

\begin{abstract}
Background/Aim: Renal fibrosis is essential for the progression of diabetic nephropathy (DN). Macrophages accumulate in diabetic kidneys and are involved in epithelial-to-mesenchymal transition (EMT), a vital mechanism leading to renal fibrosis. Recently, high-mobility group nucleosome-binding protein 1(HMGN1) was documented in promoting the recruitment and activation of antigen-presenting cells. In this study, we first reported its roles in renal fibrosis and the underlying mechanism associated with macrophage filtration and EMT. Methods: Twenty C57BL/6J mice were administered streptozotocin (STZ) to induce diabetes for 6 weeks and then divided into 4 groups: normal control group; DN group; benazepriltreated group, and insulin-treated group. Blood glucose, creatinine, and albumin in urine, hematoxylin and eosin, and Sirius red staining of kidney tissues were used to assess the renal pathology. ELISA, immunochemistry, and in situ hybridization were performed to determine the expression of HMGN1, CD68, F4/80, $\alpha$-smooth muscle actin, and E-cadherin. Results: The renal expression levels of HMGN1, macrophage markers, and EMT makers were increased in DN group, and insulin treatment could reduce the overexpression of these indicators with a better effect than benazepril treatment. Both treatments could not obviously ameliorate urine albumin-to-creatinine ratio, collagen expression, and renal histological changes in STZ-induced diabetic mice. Correlation analysis indicated that there was a relationship among HMGN1, macrophage markers, EMT markers, and collagen expression in DN mice. Conclusion: HMGN1 may promote macrophages accumulation and EMT, suggesting a potential therapeutic target for preventing renal fibrosis development in DN.
\end{abstract}




\section{Kidney \\ Blood Pressure \\ Research}

\begin{tabular}{l|l}
\hline Kidney Blood Press Res 2019:44:331-343 \\
\hline DOI: 10.1159/000499877 & $\begin{array}{l}\text { @ 2019 The Author(s). Published by S. Karger AG, Basel } \\
\text { www.karger.com/kbr }\end{array}$ \\
\hline
\end{tabular}

Yu et al.: The Role and the Potential Mechanisms of HMGN1 in DN-Associated Renal Fibrosis

\section{Introduction}

Diabetic nephropathy (DN) is the most common complication of diabetes and the leading cause of end-stage renal failure, which is a widespread occurrence presently and expected to increase in prevalence $[1,2]$. Multiple factors, including genetic background, hyperglycemia, and hemodynamic changes, have been reported to result in DN onset and progression [3]. Strategies reducing blood glucose level and complete RAS system blockade are assessed clinically as the main way to preserve renal function $[4,5]$.

The pathogenesis of DN is complicated, and the molecular mechanism remains unclear. Fibrosis is related to declining renal function in diabetic kidney disease, which is a major cause leading to end-stage renal disease (ESRD) [6, 7]. Renal fibrosis occurs when the normal tissue architecture is gradually replaced with the extracellular matrix (ECM), which has been considered as a crucial pathological change in DN. It is generally accepted that these activated myofibroblasts are the principal effector cells that are responsible for the excess deposition of interstitial ECM during renal interstitial fibrosis under pathological conditions such as high glucose [8]. Growing evidence implicated the renal tubular epithelial-to-mesenchymal transition (EMT) as an important pathway leading to the generation of interstitial myofibroblasts in the diseased kidney [9-11]. EMT occurs along with phenotypic conversion, characterized by the loss of epithelial-specific markers, such as E-cadherin, and the gain of transitional features, such as $\alpha$-smooth muscle actin $(\alpha$-SMA) $[12,13]$. However, the molecular mechanism of EMT in DN is still not fully understood.

Macrophages are found in normal kidney and in increased numbers in diseased kidney, well recognized for their pathogenic role in kidney inflammation and fibrosis $[14,15]$. Such an increase in the macrophage population has been correlated with the presence of interstitial fibrosis. Findings showed that macrophages are invariably present at sites of active renal fibrosis that contain $\alpha$-SMA-positive, matrix-producing myofibroblasts. Furthermore, the degree of macrophage infiltration correlates with both the severity of renal damage and the extent of renal fibrosis, thereby establishing a close relationship between the presence of macrophages and renal fibrosis [16]. Macrophages can secrete a number of proinflammatory cytokines including interleukin-1 (IL-1), matrix metalloproteinase-9, fibroblast growth factor-2, and transforming growth factor- $\beta$, which are capable of inducing profibrotic responses in kidney cells, including myofibroblast proliferation, ECM production, and EMT $[17,18]$. A more recent study identified that bone marrow-derived macrophages can undergo myofibroblast transition in vitro and in experimental renal fibrosis via a process of macrophage to myofibroblast transition driven by the transforming growth factor- $\beta / \operatorname{Smad} 3$ signaling pathway [19]. All these reports suggest that macrophage is an important contributor to the progression of renal fibrosis.

It is known that stimulation of the immune system via toll-like receptors (TLRs) is a common proinflammatory triggering mechanism in all forms of fibrosis [20-23]. In a murine model of pulmonary fibrosis, activated macrophages produce proinflammatory factors, such as TNF- $\alpha$ and IL- 1 that in turn activate fibroblasts and induce overproduction of ECM proteins through IL-1R1/TLR-MyD88 signaling, which is demonstrated as an essential pathway [24]. Recently, high-mobility group nucleosome-binding protein 1(HMGN1) acts as an endogenous alarmin and is capable of promoting the recruitment and activation of antigen-presenting cells via TLR4 [25-28]. Our previous study found that HMGN1 expressed in both immune cells and renal cells and increased expression of HMGN1 and TLR4 play an important role in the process of streptozotocin (STZ)-induced diabetic mice [29]. However, the molecular mechanism is poorly understood. In this study, we examined the association between HMGN1, kidney macrophage accumulation, and fibrosis, as well as its contribution to the progression of DN in STZ-treated mice. 


\section{Kidney \\ Blood Pressure \\ Research}

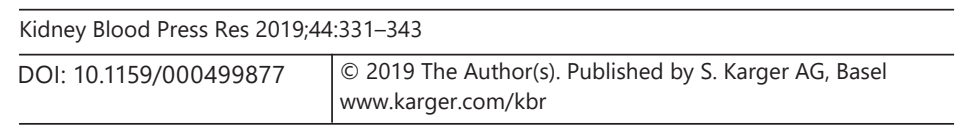

Yu et al:: The Role and the Potential Mechanisms of HMGN1 in DN-Associated Renal Fibrosis

\section{Methods}

\section{Experimental Materials}

STZ (Sigma, St. Louis, MO, USA), blood glucose meter (Sinocare Inc, Hunan, China), albumin and creatinine assay kits (Bioassay Systems, Hayward, CA, USA), insulin (Wanbang Biochemical Pharmaceutical Inc, Jiangsu, China), benazepril hydrochloride (Novartis Pharmaceutical Co. Ltd., Peking, China), hematoxylin and eosin (H\&E) staining kit, Sirius red (SR) staining kit, and bovine serum albumin (BSA; Solarbio Life Sciences, Peking, China), rabbit polyclonal anti-mouse HMGN1, CD68, F4/80, $\alpha$-SMA, and ED-1 antibody (MDL Biotech Application Co. Ltd., Peking, China), goat anti-rabbit horseradish peroxidase conjugate secondary antibody (Proteintech Group, Inc., Chicago, IL, USA), in situ hybridization (ISH) assay kit (Boster Biological Technology Co. Ltd., Wuhan, Hubei, China), and diaminobenzidine (DAB) staining kit (Zhongshan Jinqiao Biotechnology Co. Ltd., Beijing, China) were used.

\section{Animal Model and Groups}

The animal experiments were approved by the Ethics Committee of Guizhou Provincial People's Hospital. All protocols were performed in compliance with the Guide for Care and Use of Laboratory Animals as described by the PRC Ministry of Health (MoH Publication No. 55, revised 1998). Twenty 8-week-old male C57BL/6 mice with body weight ranging from 18 to $22 \mathrm{~g}$ were provided by the Experimental Animal Centre of Army Medical University. All mice were housed with a 12-h artificial light/dark cycle at $24 \pm 1^{\circ} \mathrm{C}$. After 1-week adaption, diabetes was induced by a single intraperitoneal injection of STZ $(150 \mathrm{mg} / \mathrm{kg}$ weight in citrate buffer). Likewise, the same amount of citrate buffer was administered to control animals. Tail vein blood was collected after $72 \mathrm{~h}$. Mice with a 3-day blood glucose level over $16.7 \mathrm{mmol} / \mathrm{L}$ were considered diabetic and thus included in the study.

Six weeks after the onset of diabetes, all mice were randomly divided into 4 groups as follows ( $n=5$ each group): (1) normal control group (NC group, untreated); (2) DN group (untreated); (3) benazepril-treated group (BEN group, $10 \mathrm{mg} / \mathrm{kg} /$ day i.g. infusion, diluted in $0.9 \%[\mathrm{w} / \mathrm{v}]$ normal saline); and (4) insulin-treated group (INS group, $1 \mathrm{U} /$ day i.p. injection). The treatment continued for 8 weeks, and then all mice were euthanized.

\section{Sample Collection and Testing}

After 8 weeks of treatments, mice were placed in metabolic cages for urine and sample collection. Albumin and creatinine in urine were quantified by assay kits and converted as urine albumin-to-creatinine ratio (UACR). Random blood glucose levels were determined with a drop of tail vein blood by using the glucose oxidase method on a glucose meter. The body weight of mice was measured, and all mice were then sacrificed by decapitation. The kidney from each mouse was rapidly excised, washed with saline, fixed in $10 \%(\mathrm{w} / \mathrm{v})$ formalin solution (pH 7.4) overnight, and then gradient alcohol dehydrated, transparent with xylene, and embedded in paraffin wax for histopathological examination. Kidney weight/body weight of each mouse is used as the kidney weight index.

\section{Kidney Pathology Test}

The embedded kidney tissue was cut into 2 - $\mu$ m-thick sections and processed for H\&E, SR staining for light microscopic observation. Kidney pathology was determined by glomerular and tubular morphology and inflammatory cell infiltration. Interstitial fibrosis was evaluated in SR-stained sections using Image-Pro Plus 5.1. The density of SR-stained collagen was calculated for each kidney section. Ten fields under $400 \times$ magnification were examined and average was calculated. 


\section{Kidney \\ Blood Pressure \\ Research}

\begin{tabular}{|c|c|}
\hline Kidney Blood Press Res 201 & :331-343 \\
\hline DOI: 10.1159/000499877 & $\begin{array}{l}\text { (c) } 2019 \text { The Author(s). Published by S. Karger AG, Basel } \\
\text { www.karger.com/kbr }\end{array}$ \\
\hline
\end{tabular}

Yu et al.: The Role and the Potential Mechanisms of HMGN1 in DN-Associated Renal Fibrosis

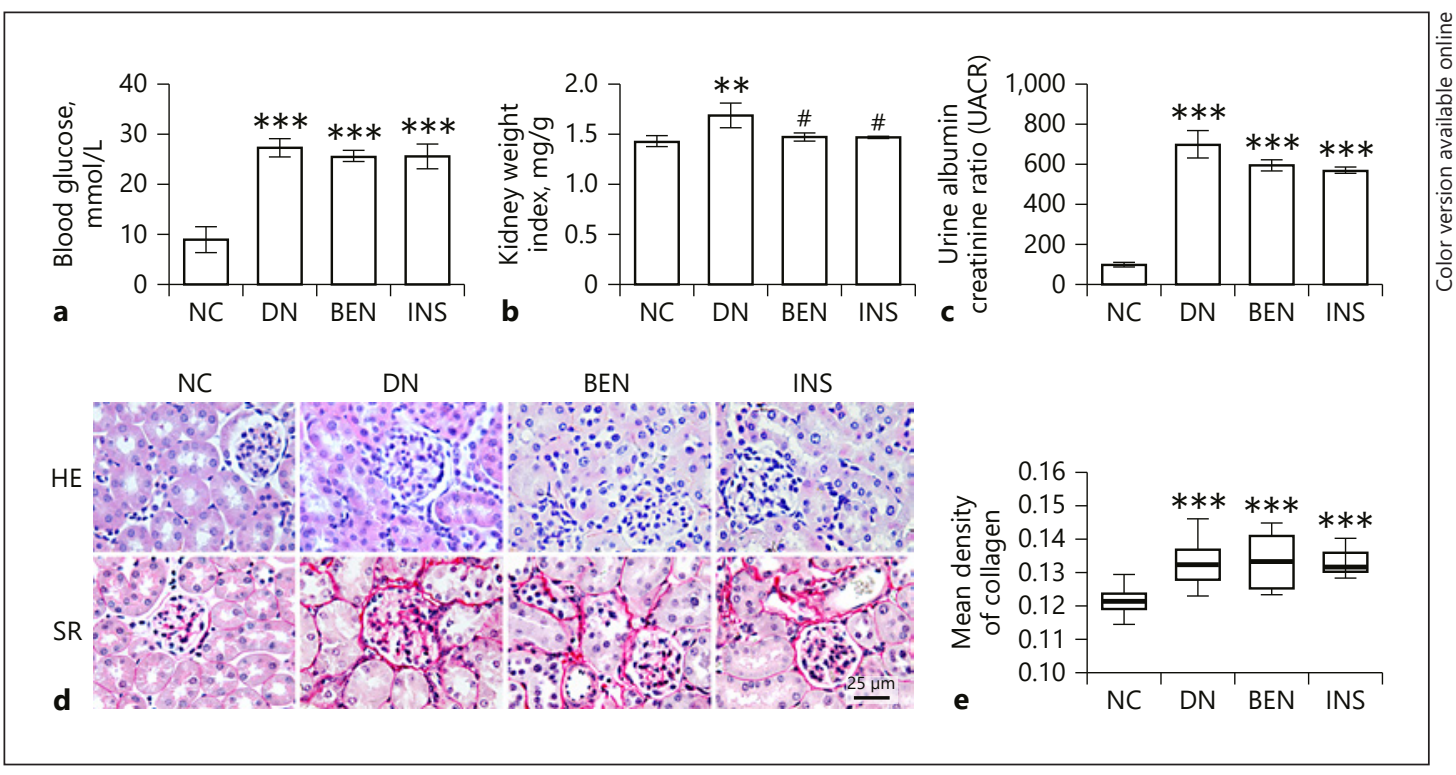

Fig. 1. The biochemical markers and representative pathological changes in renal tissue compared between groups. a Fasting blood glucose. b Kidney weight index. c Urea albumin creatinine ratio (UACR). d Renal histomorphology observed with hematoxylin and eosin staining (H\&E, 400x) and collagen of mouse kidney stained by sirius red (SR, 400x). e Quantitative analysis of collagen content in (d). Scale bars, $100 \mu \mathrm{m}$. NC, normal control mice; DN, diabetic nephropathy mice; BEN, the DN mice treated with benazepril; INS, the DN mice treated with insulin. ${ }^{* *} p<0.001,{ }^{* * *} p<0.0001$ versus NC group; ${ }^{\#} p<0.05$ versus DN group. One-way analysis of variance followed by Tukey's multiple comparison, $n=3-5$ per group.

\section{Immunohistochemical Staining}

The protein expression level in kidney tissue was detected by using immunohistochemistry (IHC). The 4- $\mu \mathrm{m}$ paraffin sections were subjected to dewaxing and rehydration, followed by antigen retrieval and $3 \%(\mathrm{w} / \mathrm{v}) \mathrm{H}_{2} \mathrm{O}_{2}$ inactivation. After washing in phosphate-buffered saline (PBS), tissue sections were blocked with $1 \%(\mathrm{w} / \mathrm{v}) \mathrm{BSA}$ in PBS and then were incubated with rabbit anti-HMGN1 antibody (1:250 dilution), CD68, F4/80, $\alpha$-SMA, and ED-1(1:200 dilution) separately overnight at $4{ }^{\circ} \mathrm{C}$. The negative control group received the antibody diluent ( $0.01 \mathrm{~mol} / \mathrm{L}$ PBS containing 1\% [w/v] BSA) only. After PBS washing (3 times, 5 min each), goat anti-rabbit IgG secondary antibody (1:200 dilution) was added to sections and incubated at $37^{\circ} \mathrm{C}$ for $1 \mathrm{~h}$. Following PBS washing ( 3 times, 5 min each), tissues were observed after DAB staining. Nuclei were stained by hematoxylin counterstaining under a light microscope. The numbers of positive staining cells in kidney were counted in 10 equivalent highpower fields (HPF $\times 400)$; positive staining was further qualified by using Image-Pro Plus 5.1 Software and expressed as mean density (IOD/area).

\section{In Situ Hybridization}

ISH was performed in paraffin sections at a thickness of $4 \mu \mathrm{m}$ to determine the HMGN1 mRNA expression level in the kidney. According to the standard procedures, the sections were prepared with deparaffin, rehydration through a graded series of ethanol baths, and then washed with $0.1 \%(\mathrm{w} / \mathrm{v}$ ) diethylpyrocarbonate-treated water for $5 \mathrm{~min} 3$ times. ISH of HMGN 1 was done at $42^{\circ} \mathrm{C}$ by using a commercial ISH kit following the manufacturer's instructions. Probes were designed, respectively, based on the published genomic sequences of mouse HMGN1 mRNA (GenBank ID: 224109), which were labeled with digoxin. The negative control group received the sense probes only. After DAB staining, renal tissue sections 


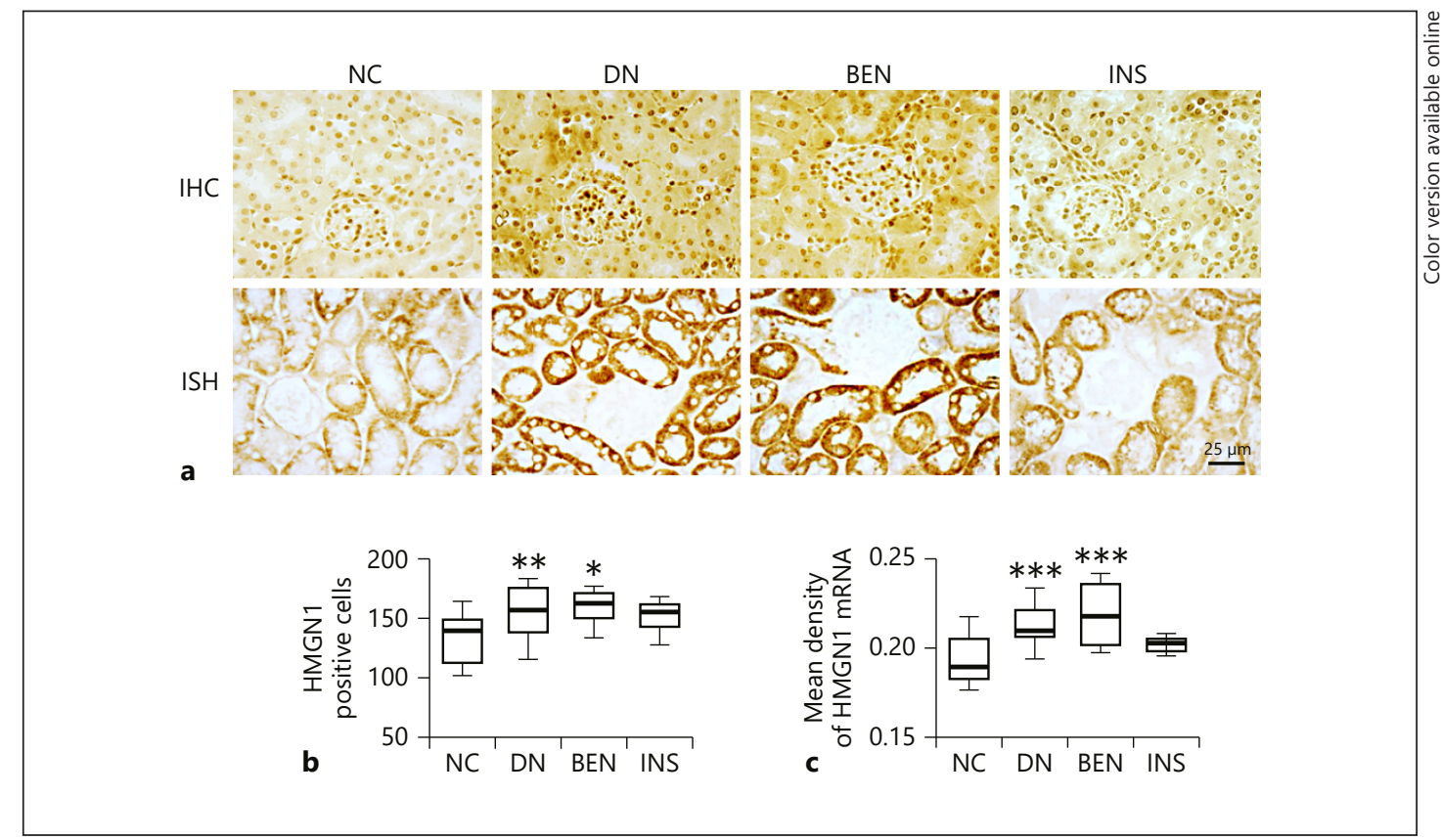

Fig. 2. The expression levels of HMGN1 protein and mRNA in mouse kidney compared between groups. a HMGN1 protein detected by immunohistochemistry (IHC, 400×) and HMGN1 mRNA determined by in situ hybridization (ISH, 400×). b Quantitative analysis of HMGN1 protein expression in (a). c Quantitative analysis of HMGN1 mRNA expression in (a). Scale bars, $100 \mu \mathrm{m}$. NC, normal control mice; DN, diabetic nephropathy mice; BEN, the DN mice treated with benazepril; INS, the DN mice treated with insulin. ${ }^{*} p<0.05$, ${ }^{* *} p<$ $0.001,{ }^{* * *} p<0.0001$ versus NC group. One-way analysis of variance followed by Tukey's multiple comparison, $n=3-5$ per group.

underwent gradient dehydration, transparency, and mounting. Tissue sections were observed and randomly photographed for 10 fields for each section. Images were analyzed by ImagePro Plus 5.1 software, and the mean density values calculated.

\section{Statistical Analysis}

All statistical data were analyzed using GraphPad Prism version 5.01. Measured data were expressed as mean \pm SD. One-way ANOVA followed by Student-Newman-Keuls posttest was used for multiple comparisons. The correlation between the 2 variables was analyzed using Pearson correlation. $p<0.05$ indicates statistically significant differences.

\section{Results}

\section{General Conditions of All Groups of Mice}

Diabetic mice model was developed with STZ induction and evaluated with blood and urine parameters. Mice with untreated DN showed severe hyperglycemia, along with a continuous decrease in renal function characterized by elevated blood glucose level, obviously increased kidney weight index, and UACR. After 8 weeks of drug treatments, blood glucose and UACR were reduced compared to diabetic mice without treatment, although still higher than that of $\mathrm{NC}$ group. The kidney weight index in 2 treatment groups was significantly lower than that in the model group. All these parameters behaved normally in nondiabetic mice (Fig. 1a-c). 


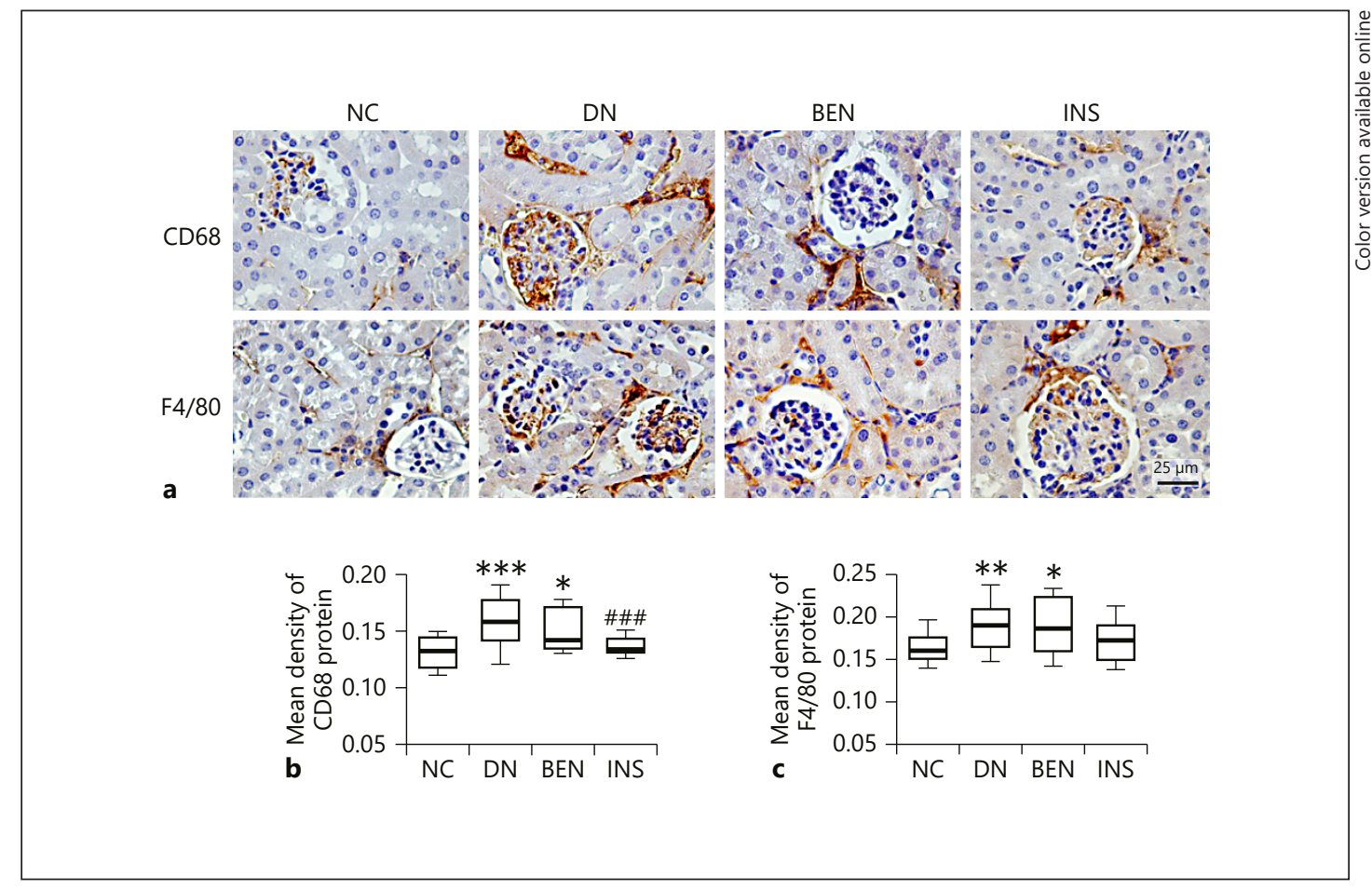

Fig. 3. The expression levels of inflammation-related protein in mouse kidney examined by IHC. a IHC staining for CD68 and F4/80 (400×). b Quantitative analysis of CD68 protein expression in (a). c Quantitative analysis of F4/80 protein expression in (a). Scale bars, $100 \mu \mathrm{m}$. NC, normal control mice; DN, diabetic nephropathy mice; BEN, the DN mice treated with benazepril; INS, the DN mice treated with insulin. ${ }^{*} p<0.05$, ${ }^{* *} p<0.001,{ }^{* * *} p<0.0001$ versus NC group; ${ }^{\# \#} p<0.0001$ versus DN group. One-way analysis of variance followed by Tukey's multiple comparison, $n=3-5$ per group.

\section{Renal Histological Changes}

The kidney histological changes were screened to confirm the results of the markers of renal functions. As shown in Figure 1, there were no obvious abnormalities in glomerular or tubular structures of the kidneys in mice of NC group. Compared with NC group, interstitial infiltration of mononuclear cells and mesangial cellularity increased and severe tubular damage occurred in the DN group, revealed by H\&E staining. SR staining and semi-quantitative analysis of collagen showed a high deposition of collagen fibers in glomerular and tubular interstitial, which were typical manifestations of renal fibrosis in the DN group. Treatment with benazepril or insulin resulted in minor improvements in these histological changes, and the statistical results for collagen in 2 treatments group remained high compared with the NC group (Fig. 1d, e).

\section{Expression Level of HMGN1 in Kidneys}

We examined the expression levels of HMGN1 protein and mRNA by IHC and ISH, respectively. Immunohistochemical staining for HMGN1 and subsequent quantitative analysis demonstrated that HMGN1 protein expression was significantly increased in the DN group, with deeper brown staining particles in the nucleus and cytoplasm, compared with the NC group. After drug administration, the HMGN1 protein expression level in BEN group remained higher than the NC group, whereas in the INS group reduced to close to normal level. The results of ISH examination showed similar results as the IHC staining (Fig. 2). 


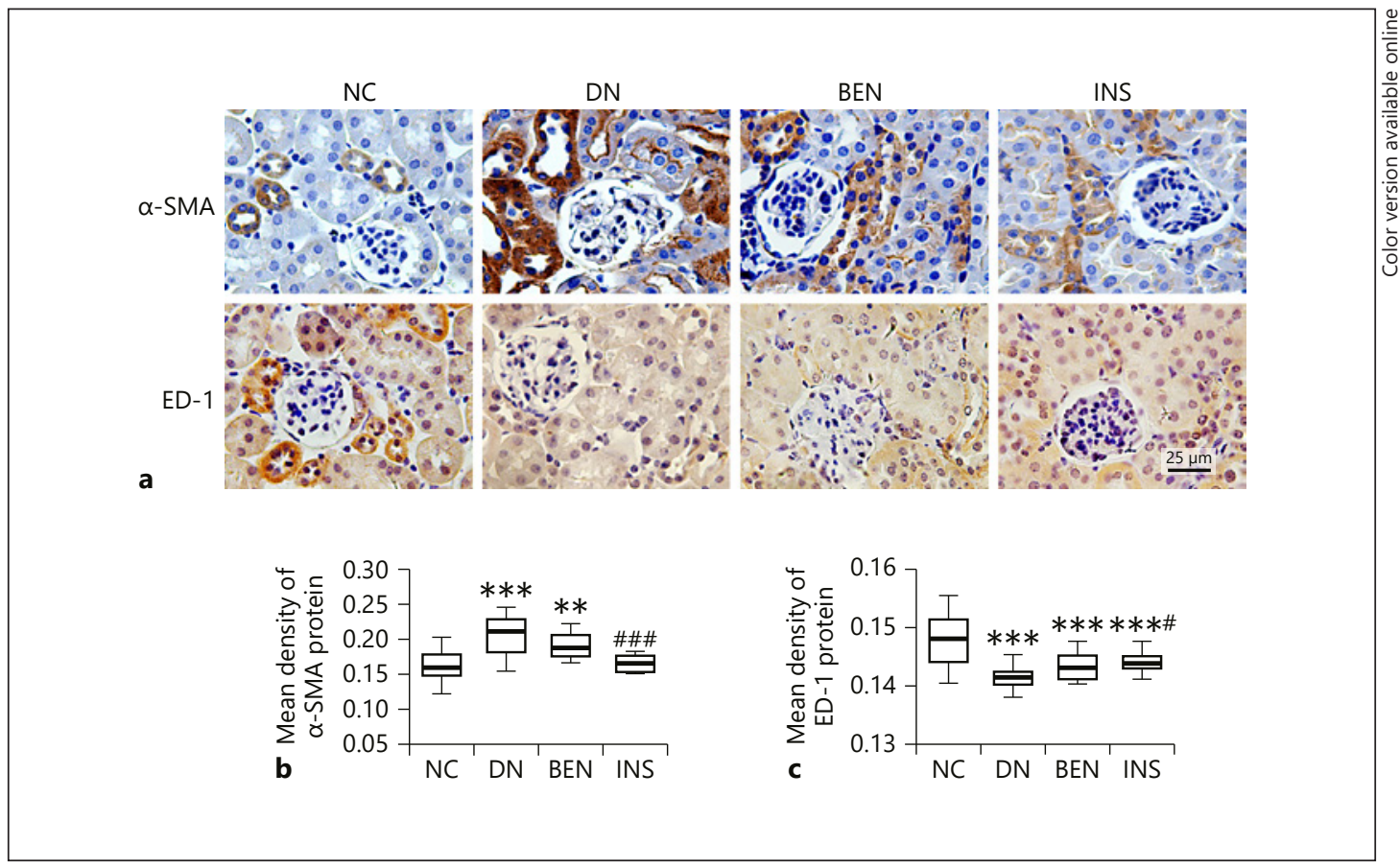

Fig. 4. The expression levels of EMT-related protein in mouse kidney examined by IHC. a IHC staining for $\alpha$-SMA and ED-1 (400×). b Quantitative analysis of $\alpha$-SMA protein expression in (a). c Quantitative analysis of ED-1 protein expression in (a). Scale bars, $100 \mu \mathrm{m}$. NC, normal control mice; DN, diabetic nephropathy mice; BEN, the DN mice treated with benazepril; INS, the DN mice treated with insulin. ${ }^{* *} p<0.001,{ }^{* * *} p<$ 0.0001 versus NC group; ${ }^{\#} p<0.05$, \#\#\# $p<0.0001$ versus DN group. One-way analysis of variance followed by Tukey's multiple comparison, $n=3-5$ per group. $\alpha$-SMA, $\alpha$-smooth muscle actin.

\section{Assessment of Inflammation in Kidneys}

We next assessed inflammatory responses in the kidneys of mice. Compared with controls, there were many little particles in the DN group, suggesting that CD68 and F4/80 protein expression has increased on the DN mice kidney tissue. The expression of CD68 and F4/80 protein in BEN group remains high. However, the brown staining of the granules was significantly reduced in the INS group, which also showed a lower mean density value for the 2 related renal inflammation factors in comparison with that of the DN group (Fig. 3a-c).

\section{Assessment of EMT in Kidneys}

Immunohistochemical staining was performed to determine the expression of EMTrelated factors. As shown in Figure 4, $\alpha$-SMA staining was more obvious in DN mice than in NC mice, while ED-1 staining was reduced. The statistical data also showed significant upregulated level of $\alpha$-SMA and downregulated level of ED-1 in DN group compared with controls. The BEN group still showed increased $\alpha$-SMA expression and reduced ED-1 expression. The $\alpha$-SMA expression was significantly suppressed, along with ED-1 expression increased obviously with insulin treatment, when compared with those of DN group (Fig. 4a-c).

\section{Correlation Analysis}

We next investigated the relationship between HMGN1, inflammation, and EMT by using correlation analysis. Before treatment, Pearson correlation analysis showed that the level of HMGN1 significantly increased in association with the changes of CD68, F4/80, 


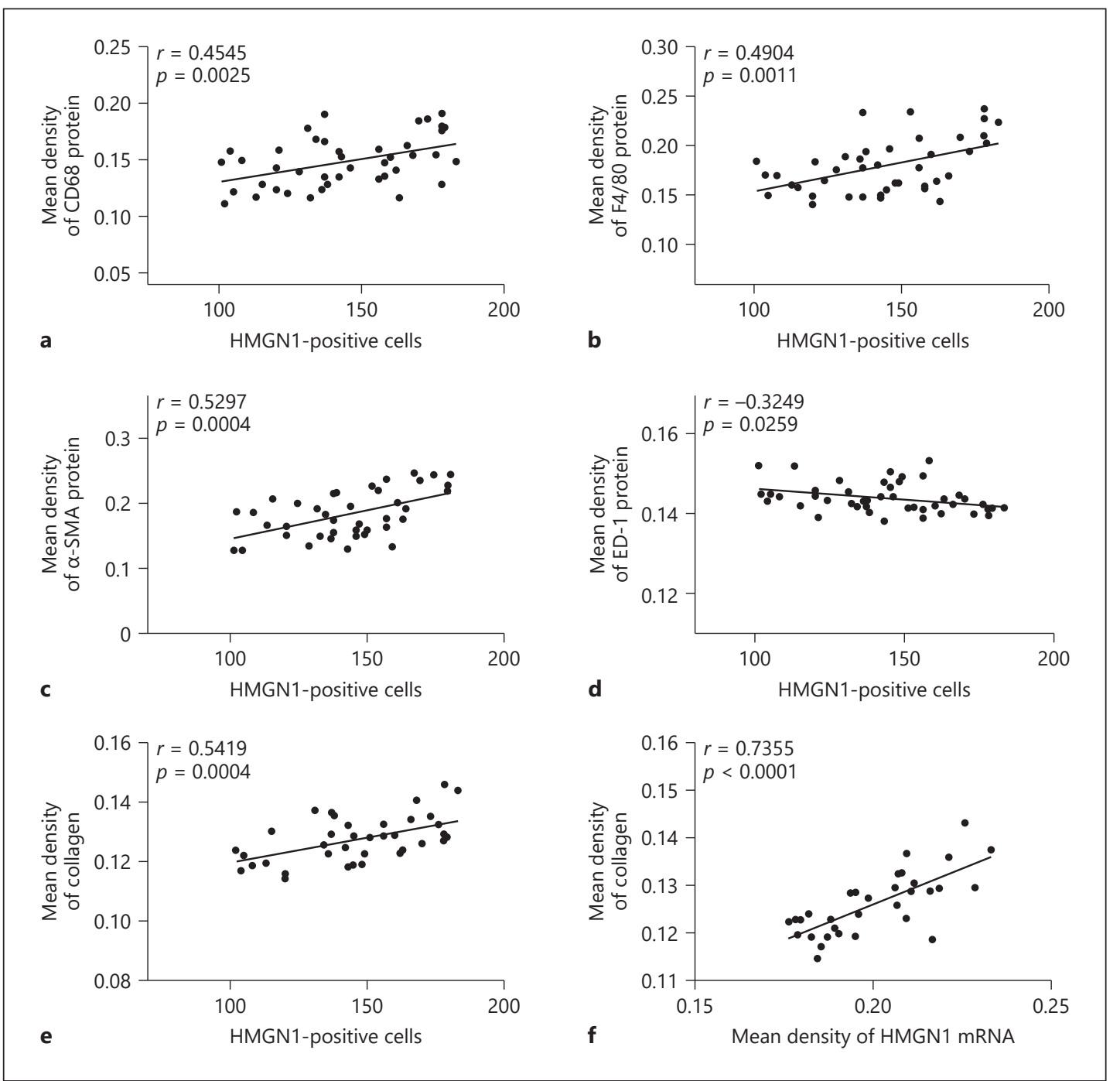

Fig. 5. The correlations between the level of HMGN1 and related molecules of inflammation, EMT, and fibrosis, respectively, in the renal tissue of DN mice before treatments. a Correlation between the expression of HMGN1 protein and CD68 protein (Spearman $r=0.4545, p=0.0025)$. b Correlation between the expression of HMGN1 protein and F4/80 protein (Spearman $r=0.4904, p=0.0011$ ). c Correlation between the expression of HMGN1 protein and $\alpha$-SMA protein (Spearman $r=0.5297, p=0.0004$ ). $\mathbf{d}$ Correlation between the expression of HMGN1 protein and ED-1 protein (Spearman $r=-0.3249, p=0.0259$ ). e Correlation between the expression of HMGN1 protein and collagen (Spearman $r=0.5419, p=0.0004$ ). $\mathbf{f}$ Correlation between the expression of HMGN1 mRNA and collagen (Spearman $r=0.7355, p<0.0001$ ). HMGN1, high-mobility group nucleosome-binding protein; $\alpha$-SMA, $\alpha$-smooth muscle actin.

$\alpha$-SMA, ED-1, and collagen deposition in mouse kidneys (Fig. 5). Inflammation-related factors (CD68 and F4/80) and fibrosis-related factors ( $\alpha$-SMA and collagen) showed a significant positive correlation (Fig. 6). After benazepril and insulin treatment, F4/80 and HMGN1 staining, $\alpha$-SMA, and inflammation-related factors (CD68 and F4/80) were significantly positively correlated (Fig. 7, 8). Interestingly, the elevated glucose level was associated with significantly upregulated HMGN1 mRNA and increased CD68 and F4/80 protein (Fig. 9a-c). 


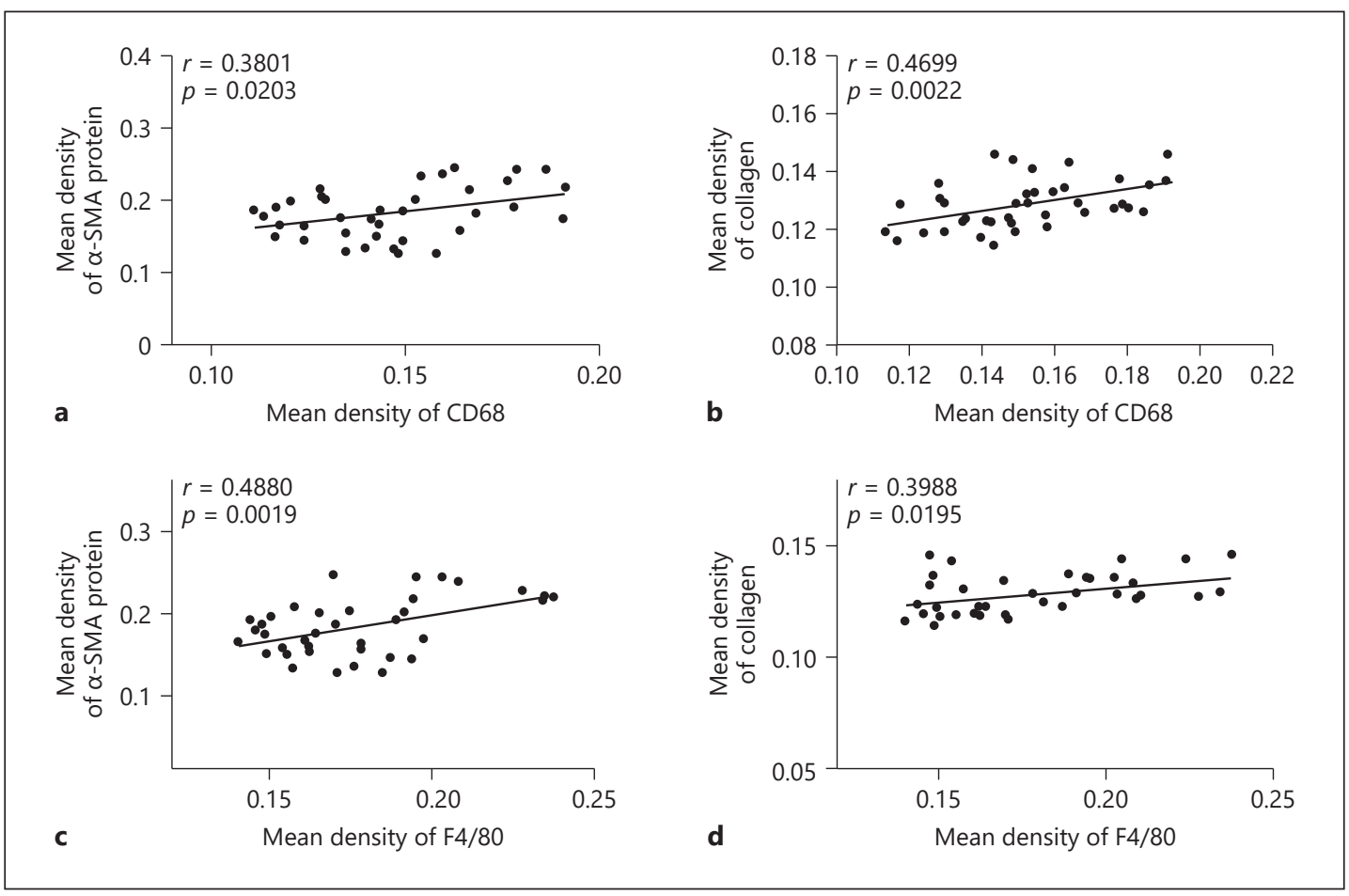

Fig. 6. The correlations among the related molecules of inflammation, EMT, and fibrosis, respectively, in the renal tissue of T2DN mice before treatments. a Correlation between the expression of CD68 protein and $\alpha$-SMA protein (Spearman $r=0.3801, p=0.0203$ ). $\mathbf{b}$ Correlation between the expression of CD68 protein and collagen (Spearman $r=0.4699, p=0.0022$ ). c Correlation between the expression of F4/80 protein and $\alpha$-SMA protein (Spearman $r=0.4880, p=0.0019$ ). $\mathbf{d}$ Correlation between the expression of $\mathrm{F} 4 / 80$ protein and collagen protein (Spearman $r=-0.3988, p=0.0195$ ). $\alpha$-SMA, $\alpha$-smooth muscle actin.

\section{Discussion}

DN is one of the most serious complications of diabetes and the primary cause of ESRD. Renal tubulointerstitial fibrosis is a crucial procession of DN toward ESRD [30]. The macrophage is well recognized for the pathogenic role in kidney inflammation and EMT, which contributes to the renal fibrosis progression [15-17]. Studies have shown that HMGN1 acts as the potential ligand of TLR4 and capable of activating and recruiting antigen-presenting cells. Its roles in DN development and the underlying mechanism associated with macrophage recruitment and EMT were first investigated in this study.

After DN model development, the mice presented typical diabetes nephropathy features, characterized by a rise in blood glucose, increase of kidney weight index, UACR, as well as fibrosis changes in renal lesions, inflammation cells infiltration, and increased collagen stained by SR. In renal kidney tissue of DN mice, the macrophage markers CD68 and F4/80 were increased, implying the macrophage accumulation. The expression level of epithelial marker E-cadherin was reduced, whereas mesenchyme phenotype $\alpha$-SMA was upregulated, indicating that EMT occurred in DN. Moreover, the expression levels of HMGN1 protein and mRNA were increased, which was in accordance with our previous findings [29]. Further analysis showed a positive correlation among HMGN1, macrophage and EMT makers, and collagen staining density. These data suggested that HMGN1 might promote the formation and development of renal fibrosis. The potential mechanism might be related to the macrophage recruitment, in addition to inducing the EMT pathway in STZ- 
Yu et al.: The Role and the Potential Mechanisms of HMGN1 in DN-Associated Renal Fibrosis

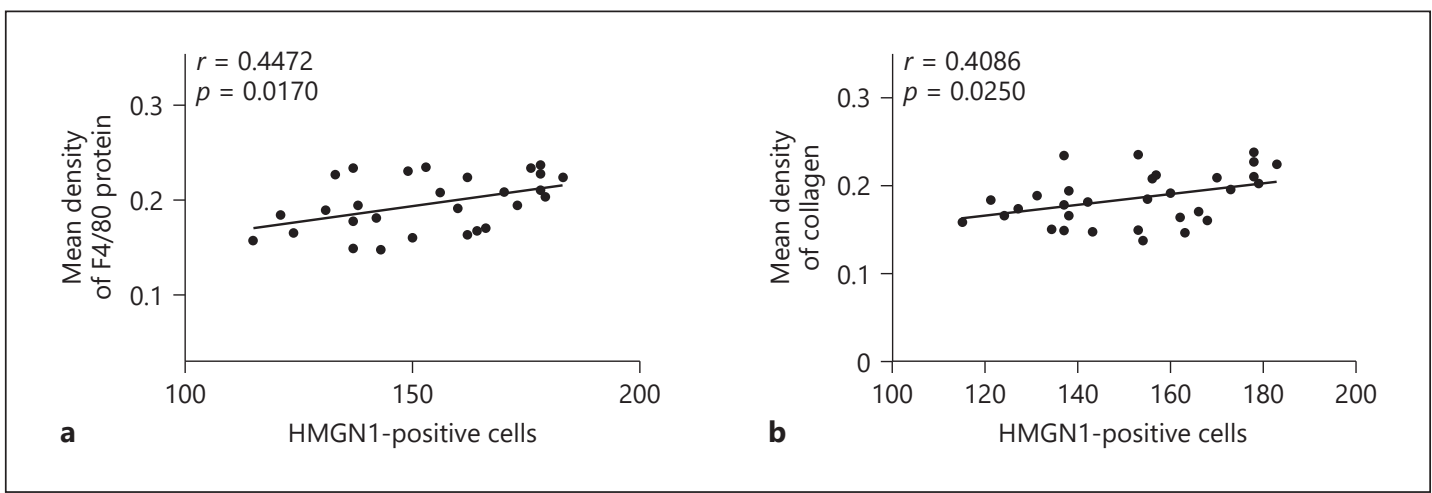

Fig. 7. The correlations between the level of HMGN1 and F4/80 in the renal tissue of DN mice after benazepril and insulin treatments, respectively. (a) Correlation between the expression of HMGN1 protein and F4/80 protein after benazepril treatment (Spearman $r=0.4472, p=0.0170$ ). (b) Correlation between the expression of HMGN1 protein and F4/80 protein after insulin treatment (Spearman $r=0.4086, p=0.0250$ ). HMGN1, high-mobility group nucleosome-binding protein.

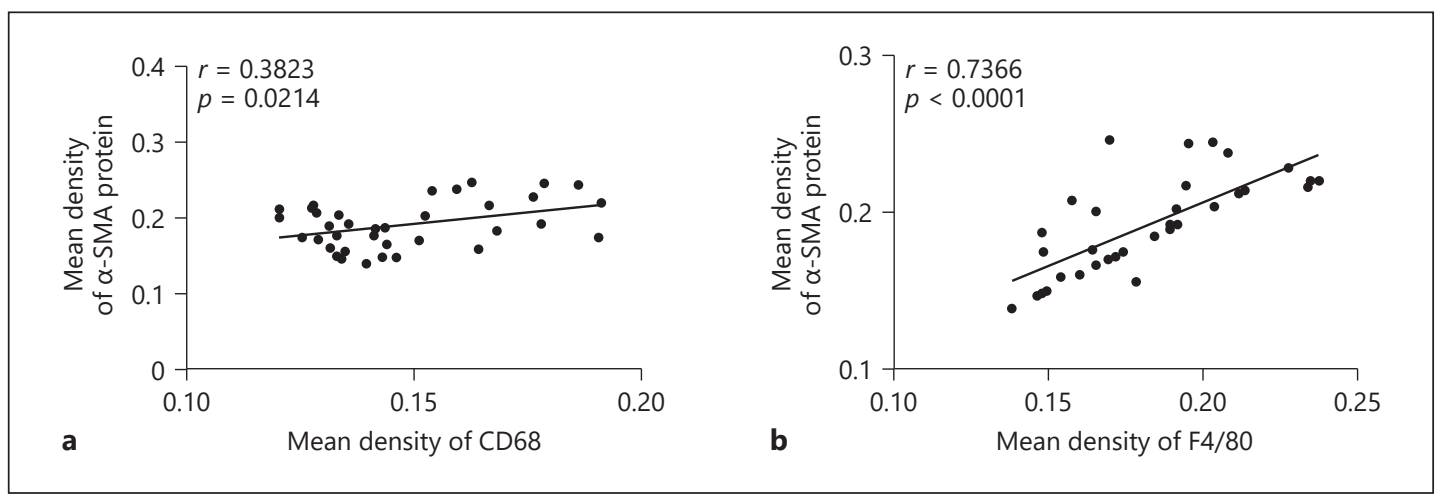

Fig. 8. The correlations between inflammation-related molecule and $\alpha$-SMA in the renal tissue of DN mice after insulin treatments. a Correlation between the expression of CD68 protein and $\alpha$-SMA protein after insulin treatment (Spearman $r=0.3823, p=0.0214$ ). $\mathbf{b}$ Correlation between the expression of F4/80 protein and $\alpha$-SMA protein after insulin treatment (Spearman $r=0.7366, p<0.0001$ ). $\alpha$-SMA, $\alpha$-smooth muscle actin.

treated mice. Our findings provided new insight into the understanding of renal fibrosis of DN.

Given the importance of HMGN1-associated pathway in renal fibrosis, we subsequently investigated the therapeutic effect of traditional intervention agents. After benazepril treatment, there was no significant change in any of the abovementioned makers. The level of F4/80 protein remained high, which still had a positive correlation with HMGN1, which suggested that HMGN1 plays a vital role in macrophage accumulation by directly TLR4mediated activation and recruitment, in addition to promoting the secretion of macrophage chemokines reported by Yang et al. [25, 27]. Conversely, the level of HMGN1, CD68, F4/80, and $\alpha$-SMA could be reduced to some extent, and E-cadherin level increased by insulin administration. Our analysis showed that the reduced level of F4/80 protein had a positive relationship with the decreased level of HMGN1. However, the collagen staining did not show a substantial change. These findings revealed that glucose controlling by insulin could suppress the macrophages infiltration induced by HMGN1 and then ameliorate EMT, but could not reverse the fibrosis outcome. Our result showed that hyperglycemia was positively corre- 

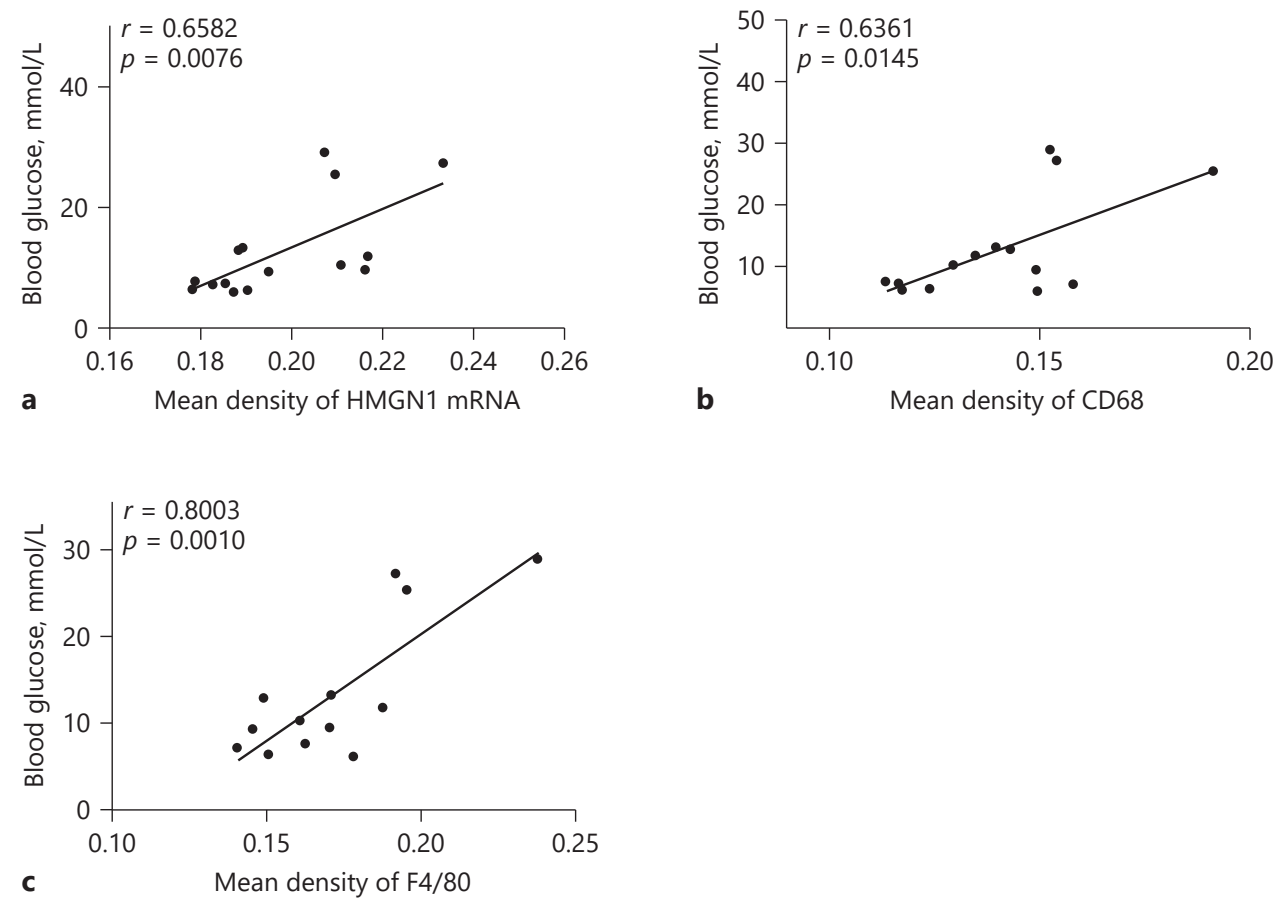

Fig. 9. The correlations among the level of blood glucose, HMGN1, and the inflammation-related molecules in DN mice before treatments. a Correlation between the expression of HMGN1 mRNA and the level of blood glucose before treatments (Spearman $r=0.6582, p=0.0076$ ). $\mathbf{b}$ Correlation between the expression of CD68 protein and the level of blood glucose before treatments (Spearman $r=0.6361, p=0.0145$ ). c Correlation between the expression of F4/80 protein and the level of blood glucose before treatments (Spearman $r=$ $0.8003, p=0.0010$ ). HMGN1, high-mobility group nucleosome-binding protein.

lated with upregulated HMGN1, CD68, and F4/80, while the reduced glucose level was not statistically significantly correlated with HMGN1, macrophage makers, EMT, and collagen level after insulin treatment. Here we proposed a hypothesis that elevated blood glucose is one factor leading to HMGN1 protein overexpression, as well as macrophage recruitment, but not the unique factor. Although more experimental evidences are needed to support this hypothesis, our results help to elucidate the reason why strategies reducing blood glucose was less effective for renal fibrosis progression or for the improvement of renal function. Our results also implies that current therapeutics for DN focusing on improving glycemic level and blood pressure control may provide little protection against renal disease progression. Developing a novel and more effective therapeutic strategy need to be investigated to prevent and reverse DN progression.

\section{Conclusion}

To conclude, this study demonstrates that HMGN1 might be involved in DN renal fibrosis occurrence and procession through the potential mechanism that is closely associated with the accumulation of macrophages and epithelial-mesenchymal transformation. Our findings provide a novel mechanism that understands renal fibrosis and also a potential therapeutic target against DN. 


\section{Kidney \\ Blood Pressure Research}

\begin{tabular}{l|l}
\hline Kidney Blood Press Res 2019:44:331-343 \\
\hline DOI: 10.1159/000499877 & $\begin{array}{l}\text { @ 2019 The Author(s). Published by S. Karger AG, Basel } \\
\text { www.karger.com/kbr }\end{array}$ \\
\hline
\end{tabular}

Yu et al.: The Role and the Potential Mechanisms of HMGN1 in DN-Associated Renal Fibrosis

\section{Acknowledgments}

This work was partly supported by the National Natural Science Foundation of China (81760134), Joint Foundation of Guizhou Provincial Science and Technology Department Guizhou Provincial People's Hospital (LH[2014]7002, LH[2015]7155, LH[2015]7154, LH[2016]7169), Science and Technology Foundation of Guizhou Province Health and Family Planning Commission (gzwkj2014-1-046), Guizhou High-Level Innovative Talents Program ([2018]5636).

\section{Disclosure Statement}

The authors report no conflicts of interest in this work.

\section{References}

1 Whiting DR, Guariguata L, Weil C, Shaw J. IDF diabetes atlas: global estimates of the prevalence of diabetes for 2011 and 2030. Diabetes Res Clin Pract. 2011 Dec;94(3):311-21.

2 Zhang L, Long J, Jiang W, Shi Y, He X, Zhou Z, et al. Trends in Chronic Kidney Disease in China. N Engl J Med. 2016 Sep;375(9):905-6.

3 Satirapoj B. Review on pathophysiology and treatment of diabetic kidney disease. J Med Assoc Thai. 2010 Nov; 93(Suppl 6):S228-41.

4 Gross JL, de Azevedo MJ, Silveiro SP, Canani LH, Caramori ML, Zelmanovitz T. Diabetic nephropathy: diagnosis, prevention, and treatment. Diabetes Care. 2005 Jan;28(1):164-76.

5 Perico N, Ruggenenti P, Remuzzi G. ACE and SGLT2 inhibitors: the future for non-diabetic and diabetic proteinuric renal disease. Curr Opin Pharmacol. 2017 Apr;33:34-40.

6 Fioretto P, Mauer M. Histopathology of diabetic nephropathy. Semin Nephrol. 2007 Mar;27(2):195-207.

7 Li J, Bertram JF. Review: endothelial-myofibroblast transition, a new player in diabetic renal fibrosis. Nephrology (Carlton). 2010 Aug;15(5):507-12.

8 Humphreys BD. Mechanisms of Renal Fibrosis. Annu Rev Physiol. 2018 Feb;80(1):309-26.

9 Loeffler I, Wolf G. Epithelial-to-Mesenchymal Transition in Diabetic Nephropathy: fact or Fiction? Cells. 2015 Oct;4(4):631-52.

10 Cruz-Solbes AS, Youker K. Epithelial to Mesenchymal Transition (EMT) and Endothelial to Mesenchymal Transition (EndMT): Role and Implications in Kidney Fibrosis. Results Probl Cell Differ. 2017;60:345-72.

11 Hills CE, Squires PE. The role of TGF- $\beta$ and epithelial-to mesenchymal transition in diabetic nephropathy. Cytokine Growth Factor Rev. 2011 Jun;22(3):131-9.

12 Li Y, Kang YS, Dai C, Kiss LP, Wen X, Liu Y. Epithelial-to-mesenchymal transition is a potential pathway leading to podocyte dysfunction and proteinuria. Am J Pathol. 2008 Feb;172(2):299-308.

13 Kang YS, Li Y, Dai C, Kiss LP, Wu C, Liu Y. Inhibition of integrin-linked kinase blocks podocyte epithelial-mesenchymal transition and ameliorates proteinuria. Kidney Int. 2010 Aug;78(4):363-73.

14 Zheng Z, Zheng F. Immune Cells and Inflammation in Diabetic Nephropathy. J Diabetes Res. 2016;2016: 1841690.

15 Cao Q, Harris DC, Wang Y. Macrophages in kidney injury, inflammation, and fibrosis. Physiology (Bethesda). 2015 May;30(3):183-94.

16 Pan B, Liu G, Jiang Z, Zheng D. Regulation of renal fibrosis by macrophage polarization. Cell Physiol Biochem. 2015;35(3):1062-9.

17 Shen B, Liu X, Fan Y, Qiu J. Macrophages regulate renal fibrosis through modulating TGF $\beta$ superfamily signaling. Inflammation. 2014 Dec;37(6):2076-84.

18 Liu Y. Epithelial to mesenchymal transition in renal fibrogenesis: pathologic significance, molecular mechanism, and therapeutic intervention. J Am Soc Nephrol. 2004 Jan;15(1):1-12.

19 Meng XM, Wang S, Huang XR, Yang C, Xiao J, Zhang Y, et al. Inflammatory macrophages can transdifferentiate into myofibroblasts during renal fibrosis. Cell Death Dis. 2016 Dec;7(12):e2495.

20 Ma J, Chadban SJ, Zhao CY, Chen X, Kwan T, Panchapakesan U, et al. TLR4 activation promotes podocyte injury and interstitial fibrosis in diabetic nephropathy. PLoS One. 2014 May;9(5):e97985.

21 Lin M, Yiu WH, Wu HJ, Chan LY, Leung JC, Au WS, et al. Toll-like receptor 4 promotes tubular inflammation in diabetic nephropathy. J Am Soc Nephrol. 2012 Jan;23(1):86-102.

22 Chen L, Sha ML, Li D, Zhu YP, Wang XJ, Jiang CY, et al. Relaxin abrogates renal interstitial fibrosis by regulating macrophage polarization via inhibition of Toll-like receptor 4 signaling. Oncotarget. 2017 Mar;8(13):2104453. 
23 Wick G, Grundtman C, Mayerl C, Wimpissinger TF, Feichtinger J, Zelger B, et al. The immunology of fibrosis. Annu Rev Immunol. 2013;31(1):107-35.

24 Gasse P, Mary C, Guenon I, Noulin N, Charron S, Schnyder-Candrian S, et al. IL-1R1/MyD88 signaling and the inflammasome are essential in pulmonary inflammation and fibrosis in mice. J Clin Invest. 2007 Dec;117(12): 3786-99.

25 Yang D, Postnikov YV, Li Y, Tewary P, de la Rosa G, Wei F, et al. High-mobility group nucleosome-binding protein 1 acts as an alarmin and is critical for lipopolysaccharide-induced immune responses. J Exp Med. 2012 Jan;209(1):157-71.

26 Wei F, Yang D, Tewary P, Li Y, Li S, Chen X, et al. The Alarmin HMGN1 contributes to antitumor immunity and is a potent immunoadjuvant. Cancer Res. 2014 Nov;74(21):5989-98.

27 Yang D, Bustin M, Oppenheim JJ. Harnessing the alarmin HMGN1 for anticancer therapy. Immunotherapy. 2015;7(11):1129-31.

28 Nie Y, Yang D, Oppenheim JJ. Alarmins and Antitumor Immunity. Clin Ther. 2016 May;38(5):1042-53.

29 Yu J, Da J, Dong R, Sun Y, Nie Y, Yu F, et al. IGF-1R Inhibitor Ameliorates Diabetic Nephropathy with Suppressed HMGN1/TLR4 Pathway. Endocr Metab Immune Disord Drug Targets. 2018;18(3):241-50.

30 Sharma D, Bhattacharya P, Kalia K, Tiwari V. Diabetic nephropathy: new insights into established therapeutic paradigms and novel molecular targets. Diabetes Res Clin Pract. 2017 Jun;128:91-108. 